\title{
A Review of Human Babesiosis and the Laboratory Diagnosis
}

\author{
Sihong Shen, Jianfa Liu* \\ School of Medicine, Ningbo University, Ningbo, China \\ Email: 760223213@qq.com, *liujianfa@nbu.edu.cn
}

How to cite this paper: Shen, S.H. and Liu, J.F. (2019) A Review of Human Babesiosis and the Laboratory Diagnosis. Open Access Library Journal, 6: e5870.

https://doi.org/10.4236/oalib.1105870

Received: October 21, 2019

Accepted: November 15, 2019

Published: November 18, 2019

Copyright $\odot 2019$ by author(s) and Open Access Library Inc.

This work is licensed under the Creative Commons Attribution International License (CC BY 4.0).

http://creativecommons.org/licenses/by/4.0/

\section{(c) () Open Access}

\begin{abstract}
Babesiosis is a new worldwide parasitic disease caused by hemoprotozoan parasites of the genus Babesia, which is parasitic on erythrocytes of humans, and other vertebrates, and transmitted via ixodid ticks. Babesiamicroti is the main pathogenic agent of human babesiosis and is prevalent all over the world, including Europe, Asia, Africa, South America, North America and Australia. The United States has the highest number of cases. Babesiosis is mainly distributed in Northeast China, followed by southwest China. As a new insect-borne infectious disease, the disease poses a serious threat to human health, but the research foundation is relatively weak and should be paid more attention to. In this paper, the prevalence, transmission, distribution of babesiosis at home and abroad, and laboratory diagnosis are reviewed. The related research focus of babesiosis mainly includes the screening of diagnostic antigens of babesiosis and the interaction mechanism between Babesia and host and other pathogens.
\end{abstract}

\section{Subject Areas \\ Epidemiology, Internal Medicine}

\section{Keywords}

Babesiosis, Tick-Borne, Babesiamicroti, Prevalence, Laboratory Diagnosis

\section{Introduction}

Babesiosis is a tick-borne hematotropic parasitic and zoonotic disease, caused by Babesia species in humans, livestock, and animals worldwide [1]. The first confirmed case of human babesiosis is reported in 1957. Subsequently, new cases of babesiosis are reported all over the world including Europe, Asia, Africa, South America, North America and Australia, while the United States still has the 
highest number of reported cases of babesiosis. The four identified babesia species definitively confirmed that infect humans so far are Babesiamicroti, $B$. divergens, B. duncani, and B. venatorum [2]. B. microti is the main etiologic agent of human babesiosis and is endemic in the northeastern and the upper Midwestern United States. After infection of Babesia, patients are usually asymptomatic or show mild malaria-like symptoms, such as chills, high fever, sweating, headaches and anorexia. Babesia can also lead to hemolytic anemia and platelet loss in patients. But in people with severe low immunity, such as splenectomized and HIV-infected patients, and people who have respiratory failure, organ dysfunction or coma, a series of fetal symptoms can easily occur and even cause death [1]. Babesiosis is diagnosed by identification of parasitemia on blood films stained with Giemsa, detection of babesia DNA by PCR, or serological rapid diagnosis in acute and convalescent sera.

\section{Transmission}

Babesia species within erythrocytes are known to be piroplasms, which can be transmitted by blood transfusion via ixodid ticks that have distinct geographical distributions based on the presence of their competent natural animal hosts, which include rodents, cattle and deer. It happens when the hosts have a tick bite or blood transfusion with infected blood, or even congenitally during pregnancy [3]. The Babesia species of human-infected babesiosis reported in China including B. venatorum and B. microti, the vectors are Haemaphysalislongicornis, Rhipicephalushaemaphysaloides, and Ixodespersulcatus. Whereas B. microti is the main species of human babesiosis in United States, followed by B. duncani. The vector is Ixodesscapularis. And the host is rodent such as deer and white-hoofed mice. In Europe, Cattle are the main hosts of babesiosis, and deer moths can also serve as storage hosts. B. divergens is the main babesia species and it is mainly transmitted by Ixodesricinus [4]. Babesiosis is globally distributed, the population is generally susceptible, and it happens more frequently in summer and autumn [5].

\section{Laboratory Diagnosis}

\subsection{Diagnosis of Pathogeny}

\subsubsection{Microscopic Examination}

The parasitemia can be detected by Microscopic examination during the acute phase of the disease, and it persists in the infected animals during the recovery phase. Blood films stained with Giemsa show better detection of parasites and clearer structure display. After staining, Up to 6 insects can be found in one erythrocyte with irregular elongated shape, and the sizes ranging from 1.5 to 1.9 $\mu \mathrm{m}$, In $B$. bovis-infected animals, parasites in peripheral blood are usually present in parasitemia below $1 \%$ of infected erythrocytes. However, in imprints or impression smears made from brain tissue parasitemia are as high as $90 \%$ of the infected erythrocytes [6]. This occurs because of the marked sequestration of 
infected erythrocytes in the microvascular beds of the brain, kidney and adrenal glands, particularly in splenectomized animals [7]. The characteristic morphology of B.bovis and B. microti is pairwise merozoites, which is difficult to distinguish under the microscope. The morphology of the early annular trophozoites of the B. microti are similar to those of the Plasmodium falciparum in red blood cells, therefore, it is easy to be misdiagnosed. The phenomenon of missed diagnosis is easy to occur due to the parasemia is generally less than $1 \%$ in the early stage of infection.

\subsubsection{Animal Inoculation and Separation}

Studies have shown that the persistence of the low parasitemia in infected animals may be detectable by the inoculation of infected blood into splenectomized animals [8]. The most sensitive animal model in lab is Mongolian gerbil,which shows acute and fetal babesiosis after the infected blood inoculation. However, animal inoculation is not suitable for diagnostic purposes, as the procedure can take weeks, so it is discouraged if other methods are available. Nevertheless, this method is not recommended for clinical diagnosis because its long cycle [9].

\subsection{Diagnosis by Immunology}

\subsubsection{Indirect Fluorescent Antibody Test (IFAT)}

IFAT is the most widely used detection technology presently. The florescent techniques and the immunological assays are both needed. BmSA1, Bm94, and Bm5-1-1 are the main antigens available for serological rapid diagnosis. However, generally speaking, the sensitivity and specificity of IFAT is not enough. For example, BmSA1, although it is highly sensitive, it is mostly used for serological diagnosis of infected animals, the antigen has not been evaluated for the diagnosis of babesiosis in human. In addition, the specific analysis shows that there is a high cross reaction between the B. bovis and B. bigemina, and also with other Plasmodium falciparum [10].

\subsubsection{Enzyme-Linked Immunosorbent Assay}

Enzyme-Linked Immunosorbent Assay (ELISA) is more specific when testing a large number of serum samples. Recently, the ELISA established by recombinant antigen for the detection of specific antibodies in serum has improved the specificity of the method and reduced the non-heterosexual binding. Generally speaking, ELISA has replaced the IFAT as the preferred diagnostic serological test in a diagnostic laboratory. A competitive ELISA (cELISA), based on the ability of serum antibody to inhibit a monoclonal antibody (Mab) binding was instrumented. In recent years, it has been found that the rod body and microsomes can release secretions during the invasion of host red blood cells, that is, the rhoptry associated protein (RAP). This conserved RAP genes have been found in several species, such as B. bovis, B. divergens, B. divaricans, B. oriental and B. gibberelli. It is suggested that the gene plays an important role in the invasion, and can also be used for species identification and classification. A purified recombinant B. bovis RAP-1 C-terminal construct was used as antigen, and the 
inhibition of binding of MAb to RAP-1 specific epitope by serum antibodies was measured to detect positive cattle [11]. Furthermore, BmSA1 could be effectively detected by double antibody sandwich ELISA in the early stage of infection of hamster with $100 \%$ specificity, and the minimum detection limit was $2.5 \mathrm{ng} / \mathrm{ml}$. BmSA1 is one of the best diagnostic antigens in the literature [12].

\subsubsection{Immunochromatography}

Serological tests have several restrictions for the diagnosis of bovine babesiosis, as laboratory materials, appropriate equipment and trained personnel are usually required, in addition to being labor intensive and time-consuming. Those limitations apparently are solved in part by the development of the immunochromatographic test (ICT), also known as lateral flow test or strip test. The ICT is a nitrocellulose membrane-based immunoassay which does not require any instrument, is rapid and sensitive. In addition, it has a great advantage for use in clinical and field application directly in the farms. An evident advantage is that results are provided in less than $15 \mathrm{~min}$. [13] Another study, using an ICT to detect antibodies of B. bovis and B. bigemina both individually and in a dual ICT using serum from bovine samples collected in the field, showed a kappa coefficient $>0.7$ when compared to the ELISA [14].

\subsubsection{Immunohistochemical Assay}

The basic principle of the immunohistochemical assay is that the specific antibody labeled with the color-developing agent is in-situ combined with the antigen in infected animals, after the coloration, the antigen in the tissue is positioned. Researchers use this method to detect formalin-fixed and paraffin-embedded tissue and blood smears derived from $12 \mathrm{~B}$. microti-infected monkeys and 7 B. microti-infected patients. Results indicate B.microti is detectable in the red blood cells in the heart, spleen, liver and blood smear of the monkeys and the human, showing a potential technique for the diagnosis and the pathogenesis of the babesiosis.

\subsection{Diagnosis of Molecular Biology}

\subsubsection{Polymerase Chain Reaction (PCR)}

Babesial infections are difficult to detect because of the low number of parasites in peripheral blood. Therefore, DNA-based molecular methods have been developed with great advantages, such as high analytical sensitivity and specificity rates. Analytical sensitivity assays showed detection of as low as $100 \mathrm{fg}$ of parasite $18 \mathrm{~s}$ rDNA equivalent to $0.0000001 \%$ of infected erythrocytes. A colorimetric B. bigemina DNA probe was utilized in an epidemiological survey, which allowed to detect parasitemias as low as $0.001 \%$. Thus, this assay turned out good to detect asymptomatic carriers in the field [15].

\subsubsection{Nested-PCR}

Nested-PCR is a two-round amplification reaction with two pairs of primers on the inner and outer sides, so the target fragment can be amplified from a trace 
template, which further improves the sensitivity and specificity of detection compared with ordinary PCR. A heterotrophic nested-PCR is constructed to not only detect the Babesia effectively, but also sequence the amplification products. RAP-1, spherical body protein 2, and spherical body protein 4 of B. bovis have confirmed to be an effective tool for epidemiological investigations in healthy, persistently infected carriers [16] [17]. Nonetheless, the higher false positive is caused due to the sample can be easily polluted during the two-round amplification.

\subsubsection{Real-Time PCR}

Real-Time PCR is a method which combines fluorescence labeling probe technique with PCR technique to quantify parasite nucleic acid. The quantity of amplified product is measured in real time by continuously monitoring the change of fluorescence signal intensity during exponential amplification. Less amount of the initial template is required for this method comparing to conventional PCR. The disadvantage is that it is not suitable for ordinary laboratory, as it needs expensive fluorescence microscope and higher expertise skills. A quantitative PCR (qPCR) used for field surveys is discovered. Findings verify that qPCR is more sensitive than direct microscopy ( $100 \%$ vs. $10 \%$ positivity, respectively) in the detection of B. bovis.

\subsubsection{Loop-Mediated Isothermal Amplification (LAMP)}

The amplification of DNA can be synchronized with the detection of products using LAMP and there is no need for expensive equipment or complex operation step. The amplification results can be observed directly with the naked eye, which is very suitable for the promotion of ordinary laboratories. LAMP allows detection and discriminates between B. bovis and B. bigemina species with high sensitivity [18]. Therefore, the establishment of LAMP diagnostic technology for babesiosis will have a great application prospect, especially for the identification of carrier animals with very low parasitaemia.

\section{Babesiosis in the World}

Infections with Babesia species generally follow regional distributions. The United States has the largest number of reported cases of babesiosis in the world, especially in the northeastern coastal areas of Massachusetts, New York, Connecticut and Rhode Island, which are the main prevalent areas. Data released by the Centers for Disease Control and Prevention in 2016 shows that 5542 suspected and confirmed cases of babesiosis occurred in the United States from 2011 to 2014. 82\% cases occurred in June to August [19].

The first case of babesiosis is reported in France in 1976. Up to now, more than 60 cases of babesiosis have been reported in Europe, of which nearly 50 cases were reported before 2013 and more than 10 cases after 2013. Although the total number of Babesiosis cases is not large, there is a trend of sporadic and diverse species. The cases were mainly distributed in Italy, Germany and Sweden 
[20].

Africa is a severe malaria prevalent area, babesiosis is easy to be misdiagnosed or missed because Babesia species and Plasmodium are similar in shape in the red stage. Nearly 30 cases of Babesia infection have been reported in Africa, which diversely distributed in Tanzania, Mozambique, Egypt, and South Africa. The species of babesiosis in human has not been completely determined [21].

The babesiosis cases in Australia and Asia is sporadic and the Babesia species are multiple.

\section{Babesiosis in China}

Babesiosis is rarely reported in China. In 1982, Li Jinfu discovered the first case of human babesiosis in Yunnan Province. So far, more than 150 cases of human babesiosis have been reported in China, which are widely distributed in north-west, north-east, eastern China, Southern China, and southwestern provinces. The province with the highest incidence of Babesia infection in China is Heilongjiang Province in the north-east region. A total of 120 cases of babesiosis or hidden infection have been reported in this province. The second biggest epidemic regions are Chongqing and Yunnan Province with more than 20 cases are reported. A total of 6 cases are reported in eastern China. There are various species of Babesia infection, which include B. divergens, B. microti, Babesia sp. $X X B /$ Hang Zhou and other unidentified species [22].

\section{Summary}

Babesiosis is an important zoonotic disease distributed all over the world. Babesia was first found in bovine red blood cells. In the middle of the 20th century, human cases of babesiosis were diagnosed in China and Yugoslavia subsequently. The United States is the country with the largest number of reported cases in the world. Although Chinese researchers have previously described the characteristics of babesiosis, the researches on babesiosis are focused on the field of veterinary medicine, while less work has been carried out on the screening and related scientific research of babesiosis in population. Presently, the mechanism of invasion of host cells and the induction of host immune response need to be further studied. In the future, we can use bioinformatics to predict and analyze the database of babesiosis based on genomics, proteomics, metabolomics, etc. In addition, high throughput screening system of protein chip is capable of detecting potential pathogenic molecules, analyzing the interaction between host and parasites, evaluating their potentiality as new diagnostic antigen, invasion target and vaccine design potential, so as to provide reference for screening the field diagnosis of babesiosis and developing the vaccine.

\section{Conflicts of Interest}

The authors declare no conflicts of interest regarding the publication of this paper. 


\section{References}

[1] Vannier, E. and Krause, P.J. (2012) Human Babesiosis. The New England Journal of Medicine, 366, 2397-2407. https://doi.org/10.1056/nejmra1202018

[2] Jiang, J.F., et al. (2015) Epidemiological, Clinical, and Laboratory Characteristics of 48 Cases of "Babesia Venatorum" Infection in China: A Descriptive Study. The Lancet Infectious Diseases, 15, 196-203. https://doi.org/10.1016/S1473-3099(14)71046-1

[3] Leiby, D.A. (2011) Transfusion-Associated Babesiosis: Shouldn't We Be Ticked off? Annals of Internal Medicine, 155, 556-557. https://doi.org/10.7326/0003-4819-155-8-201110180-00363

[4] Homer, M.J., Aguilar-Delfin, I., Telford, S.R., Krause, P.J. and Persing, D.H. (2000) Babesiosis. Clinical Microbiology Reviews, 13, 451-469. https://doi.org/10.1128/CMR.13.3.451-469.2000

[5] Ord, R.L. and Lobo, C.A. (2015) Human Babesiosis: Pathogens, Prevalence, Diagnosis and Treatment. Current Clinical Microbiology Reports, 2, 173-181. https://doi.org/10.1007/s40588-015-0025-Z

[6] Blevins, S.M., Greenfield, R.A. and Bronze, M.S. (2008) Blood Smear Analysis in Babesiosis, Ehrlichiosis, Relapsing Fever, Malaria, and Chagas Disease. Cleveland Clinic Journal of Medicine, 75, 521-530.

[7] Everitt, J.I., Shadduck, J.A., Steinkamp, C. and Clabaugh, G. (1986) Experimental Babesia bovis Infection in Holstein Calves. Veterinary Pathology, 23, 556-562. https://doi.org/10.1177/030098588602300503

[8] Holman, P.J., et al. (2005) Comparative Infectivity of Babesia Divergens and a Zoonotic Babesia Divergens-Like Parasite in Cattle. The American Journal of Tropical Medicine and Hygiene, 73, 865-870. https://doi.org/10.4269/ajtmh.2005.73.865

[9] Dkhil, M.A., Al-Quraishy, S. and Al-Khalifa, M.S. (2014) The Effect of Babesia divergens Infection on the Spleen of Mongolian Gerbils. BioMed Research International, 2014, Article ID: 483854. https://doi.org/10.1155/2014/483854

[10] Maharana, B.R., Tewari, A.K., Saravanan, B.C. and Sudhakar, N.R. (2016) Important Hemoprotozoan Diseases of Livestock: Challenges in Current Diagnostics and Therapeutics: An Update. Veterinary World, 9, 487-495. https://doi.org/10.14202/vetworld.2016.487-495

[11] Goff, W.L., et al. (2003) Competitive Enzyme-Linked Immunosorbent Assay Based on a Rhoptry-Associated Protein 1 Epitope Specifically Identifies Babesia bovis-Infected Cattle. Clinical and Diagnostic Laboratory Immunology, 10, 38-43. https://doi.org/10.1128/CDLI.10.1.38-43.2003

[12] Priest, J.W., et al. (2012) Multiplex Assay Detection of Immunoglobulin G Antibodies that Recognize Babesia microti Antigens. Clinical and Vaccine Immunology, 19, 1539-1548. https://doi.org/10.1128/CVI.00313-12

[13] Rhemrev-Boom, M.M., Yates, M., Rudolph, M. and Raedts, M. (2001) (Immuno)affinity Chromatography: A Versatile Tool for Fast and Selective Purification, Concentration, Isolation and Analysis. Journal of Pharmaceutical and Biomedical Analysis, 24, 825-833. https://doi.org/10.1016/S0731-7085(00)00549-5

[14] Guswanto, A., et al. (2017) Evaluation of Immunochromatographic Test (ICT) strips for the Serological Detection of Babesia bovis and Babesia bigemina Infection in Cattle from Western Java, Indonesia. Veterinary Parasitology, 239, 76-79. https://doi.org/10.1016/j.vetpar.2017.04.010

[15] Criado-Fornelio, A. (2007) A Review of Nucleic-Acid-Based Diagnostic Tests for 
Babesia and Theileria, with Emphasis on Bovine Piroplasms. Parassitologia, 49, 39-44.

[16] Alvarez, J.A., Rojas, C. and Figueroa, J.V. (2019) Diagnostic Tools for the Identification of Babesia sp. in Persistently Infected Cattle. Pathogens, 8, 143. https://doi.org/10.3390/pathogens8030143

[17] El-Sayed, S., Rizk, M.A., Terkawi, M. and Igarashi, I. (2019) Cocktail Babesia bovis Antigens for Global Detection of Babesia bovis Infection in Cattle. Experimental Parasitology, 206, Article ID: 107758. https://doi.org/10.1016/j.exppara.2019.107758

[18] Liu, A., et al. (2012) Loop-Mediated Isothermal Amplification (LAMP) Method Based on Two Species-Specific Primer Sets for the Rapid Identification of Chinese Babesia bovis and B. bigemina. Parasitology International, 61, 658-663.

https://doi.org/10.1016/j.parint.2012.07.004

[19] Herwaldt, B.L., et al. (2004) Babesia divergens-Like Infection, Washington State. Emerging Infectious Diseases Journal, 10, 622-629. https://doi.org/10.3201/eid1004.030377

[20] Solano-Gallego, L., Sainz, Á., Roura, X., Estrada-Peña, A. and Miró, G. (2016) A Review of Canine Babesiosis: The European Perspective. Parasites \& Vectors, 9, 336-336. https://doi.org/10.1186/s13071-016-1596-0

[21] Bloch, E.M., et al. (2018) Babesia microti and Malaria Infection in Africa: A Pilot Serosurvey in Kilosa District, Tanzania. The American Journal of Tropical Medicine and Hygiene, 99, 51-56. https://doi.org/10.4269/ajtmh.18-0012

[22] Zhou, X., et al. (2014) Human Babesiosis, an Emerging Tick-Borne Disease in the People's Republic of China. Parasites \& Vectors, 7, 509-509.

https://doi.org/10.1186/s13071-014-0509-3 